\title{
Editorial
}

\section{Brauchen wir eine Deutsche Gesellschaft für Public health?}

Nach Jahrzehnten der Stagnation kann in Deutschland von einer eindrucksvollen Renaissance der „Öffentlichen Gesundheit" gesprochen werden. Überraschend schnell haben sich innerhalb weniger Jahre die $\mathrm{Ge}$ sundheitswissenschaften an den deutschen Universitäten (derzeit 8) etabliert. Anfang Oktober hat in Dresden der erste zweitägige Kongreß der 5 Forschungsverbünde Public health stattgefunden, kurz zuvor die zweite gemeinsame Jahrestagung der sozialmedizinischen, medizinsoziologischen und epidemiologischen Vereinigungen in Magdeburg. Aber es gibt auch noch die Medizininformatiker, die Biometriker und eine weitere epidemiologische Gesellschaft sowie die Verbände der einschlägigen Berufsgruppen. Schließlich bieten neben den universitären Public-health-Standorten inzwischen auch Fachhochschulen Studiengänge etwa zur Gesundheitsförderung oder für Pflegedidaktik bzw. Pflegemanagement an. Da sind die unterschiedlichen Benennungen der Programme und Vereine nur Indikator eines Kommunikations- und Integrationsdefizits, das für die ersten Jahre einer nachholenden Entwicklung toleriert, aber nicht auf Dauer akzeptiert werden sollte. Die Termini reichen von der Bevölkerungsmedizin über die öffentliche Gesundheit bis zu den Gesundheitswissenschaften.

Am Rande: Worte werden oft stellvertretend für ihren Mißbrauch geächtet, sonst könnten wir uns anstelle von Public health den alten deutschen Begriff von der Volksgesundheit wiederaneignen! Sein angelsächsisches Pendant "population health" bringt das eigentliche Ziel von Public health auf den Punkt.

Trotz aller Erfolgsmeldungen und aller Selbstbestätigung handelt es sich aber um einen randständigen Sektor. Beleg: die Einigung der Europäischen Unionsstaaten auf ein Kommissionsmandat für Public health $(\$ 129 \mathrm{im}$ Vertrag von Maastricht); einen solchen Auftrag hätte es für die kurative Versorgung gerade wegen ihrer politischen und wirtschaftlichen Bedeutung im nationalen Rahmen nie gegeben. Die realistische Einschätzung dieser Situation hat - parallel zum Maastricht-Proze $\beta$-, zur Gründung der European Public Health Association (EUPHA) geführt mit dem Slogan: eine Gesellschaft, ein Journal, eine Tagung. Ist das Modell für Deutschland so falsch? Sicher müßten einige Besitzstände zur Disposition gestellt werden, aber der Einfluß würde doch enorm wachsen. Als Beispiel mag die American Public Health Association dienen, deren großes Gewicht in fast jeder Kongreßanhörung zu Gesundheitsfragen durchschlägt. Bei uns steht jedoch neben kooperativen Tendenzen (siehe der o. e. Magdeburger Kongre $B$ ) auch ein legitimes Bemühen um Selbstfindung und Selbstbestimmung in den Subdisziplinen und einzelnen Berufsgruppen. So wird z. B. von einigen Epidemiologen die Meinung vertreten, sie sollten eine zweite unabhängige epidemiologische Gesellschaft in Deutschland gründen. Erwartete Größen- 
ordnung: wenige hundert Mitglieder; die meisten deutschen Gruppierungen mit Public-health-Bezug liegen deutlich unter 1000.

Im wesentlichen stehen zwei Modelle zur Verfügung, um heterogene Gruppierungen mit in Teilen auch gegenläufigen Bestrebungen zusammenzuführen bzw. zusammenzuhalten: ein Konsortialmodell und ein Sektionsmodell. Beide schließen sich nicht etwa aus, sondern können sich ergänzen. Die Einrichtung von Sektionen bietet sich an, um innerhalb einer Gesellschaft Raum für neue Entwicklungen und Querverbindungen zu schaffen. Im Konsortium finden sich eigenständige Institutionen oder Vereinigungen zusammen, um gemeinsame Projekte (z. B. Zeitschrift, Kongreß) oder Interessen (z. B. Forschungsförderung) voranzubringen, oft mit der Tendenz, zum richtigen Zeitpunkt gemeinsame Entscheidungsgremien/Vorstände zu bilden.

Ein solcher Integrationsproze $\beta$ kann nur gelingen, wenn sorgfältig auf Transparenz geachtet und von Anfang an alle, die es wollen, einbezogen werden. $\mathrm{Bi}$ - und trilaterale Absprachen tragen nur zur Polarisierung bei: die öffentliche Gesundheit wird dann zum hin- und hergezerrten Kadaver zwischen konkurrierenden Ansprüchen. Dies betrifft v. a. auch das Spannungsverhältnis zwischen den Berufsverbänden im Gesundheitswesen und den wissenschaftlichen Gesellschaften und Hochschulverbünden. Anwendungsorientierung und Praxisrelevanz sind für die Gesundheitswissenschaften langfristig, d. h. über die geförderte Startphase hinaus, von vitalem Interesse; nur eine Verwissenschaftlichung kann etwa für den öffentlichen Gesundheitsdienst noch Überlebenschancen eröffnen -, und vielleicht gilt dies auch für die kassenärztliche Primärversorgung. In einigen Landesverbänden der Ärzte des öffentlichen Gesundheitsdienstes wird eine berufsübergreifende Ausweitung der Verbandsarbeit erwogen. Sollten diese Entwicklungen nicht sinnvoll miteinander verbunden werden können? Der Deutsche Verband für Gesundheitswissenschaften (DVGE) verfolgt satzungsgemäß sowohl berufspolitische wie gesundheitswissenschaftliche Ziele. Wenn alle sich in die gleiche Richtung bewegen, warum dann nicht gemeinsam?

Zumindest für eine wirksame Vertretung auf der europäischen und internationalen Ebene wäre dies ein Gewinn! Dort wird die deutsche Zersplitterung je nachdem mit Bedauern oder Herablassung zur Kenntnis genommen. Kleinere Länder wie Schweden oder die Niederlande sind oft qualitativ und sogar quantitativ besser vertreten. Wir sollten darüber ernsthaft nachdenken!

Ulrich Laaser 